\title{
A novel rodent Chapparvovirus in feces of wild rats
}

\author{
Shixing Yang ${ }^{1 \dagger}$, Zhijian $\mathrm{Liu}^{1+}$, Yan Wang ${ }^{1}$, Wang Li ${ }^{1}$, Xingli Fu', Yuan Lin², Quan Shen ${ }^{1}$, Xiaochun Wang ${ }^{1}$, \\ Hua Wang ${ }^{1}$ and Wen Zhang ${ }^{1,3^{*}}$
}

\begin{abstract}
Chapparvovirus, a recently determined new genus in the family Parvoviridae, can infect many species of animals including bats, chickens, and pigs. Here, using viral metagenomics method, we identified a novel Chapparvovirus from feces of wild rats and designated it as rat parvovirus 2 (RPV2). The nearly complete genome of RPV2 is 4222-nt long and includes two ORFs encoding a 654-aa nonstructural protein 1 (NS1) and a 472-aa capsid protein (VP), respectively. Phylogenetic analysis over the amino acid sequence of the NS1 showed that RPV2 clustered with Eidolon helvum parvovirus 2 (EHPV2), porcine parvovirus 7 (PPV7), and turkey parvovirus 1 (TP1), forming a separate clade. Sequence analysis indicated that the NS1 protein of RPV2 shared the highest amino acid sequence identity (51\%) with that of EHPV2. According to the genetic distance-based criteria, RPV2 identified here belongs to a novel species of Chapparvovirus.
\end{abstract}

Keywords: Wild rat, Chapparvovirus, Genome structure, Viral Metagenomics

\section{Findings}

Parvoviruses can infect many species of animals and cause illness through individual or together with other viruses [1-3]. The virion of parvovirus is icosahedral and non-enveloped package, whose core is a linear single-stranded DNA genome of approximately $5 \mathrm{~Kb}$ long. The family Parvoviridae is divided into the subfamilies Parvovirinae and Densovirinae [4], which infect vertebrates and invertebrates, respectively. The subfamily Parvovirinae is divided into 8 genera: Dependoparvovirus, Copiparvovirus, Bocaparvovirus, Amdoparvovirus, Aveparvovirus, Protoparvovirus, Tetraparvovirus, and Erythroparvovirus. Recently two other genera of Marinoparvovirus and Chapparvovirus were identified. The genus of Marinoparvovirus only has one member named sesavirus which was discovered from the feces of a California sea lion pup suffering from malnutrition and pneumonia [5]. The genus of Chapparvovirus have been detected from chicken feces samples, Eidolon helvum

\footnotetext{
* Correspondence: z0216wen@yahoo.com

${ }^{\dagger}$ Equal contributors

'School of Medicine, Jiangsu University, Zhenjiang, Jiangsu 212013, People's Republic of China

${ }^{3}$ School of Medicine, Jiangsu University, 301 Xuefu Road, Zhenjiang, Jiangsu 200240, People's Republic of China

Full list of author information is available at the end of the article
}

feces samples, and porcine multiple tissue samples including serum, rectal swab, nasal swab, and lung lavage [6-8]. Chapparvovirus, as a novel genus of parvovirinae, still have unclear species. Here, using viral metagenomics and PCR amplification, we identified a novel species of Chapparvovirus from the feces of wild rats and characterized its nearly complete genome.

In the present study, we investigated 40 fecal samples from wild rats captured by the Chinese Center for Disease Control and Prevention in Taizhou City from three districts of Taizhou City including Taixing $(n=15)$, Gaogang $(n=15)$, and Hailing $(n=10)$ from June to August in 2004. All of the wild rats were adults and the exact age was unknown.

Viral metagenomics method was used to characterize viral sequences in the fecal samples. Fecal samples were suspended in DPBS, vortexed for $10 \mathrm{~min}$, and then centrifuged at $12,000 \times \mathrm{g}$ for $10 \mathrm{~min}$. The stool suspensions were collected in $1.5 \mathrm{ml}$ centrifuge tubes. Three pools were randomly generated, each of which contained 10 fecal suspensions. After low speed centrifugation and filtration, the samples were treated with DNase and RNase, to reduce levels of mink nucleic acids while viral genomes are protected from digestion within viral capsid $[9,10]$. Three libraries were then constructed using 
a

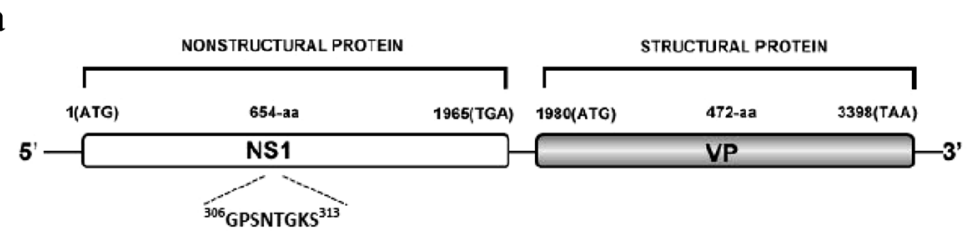

b

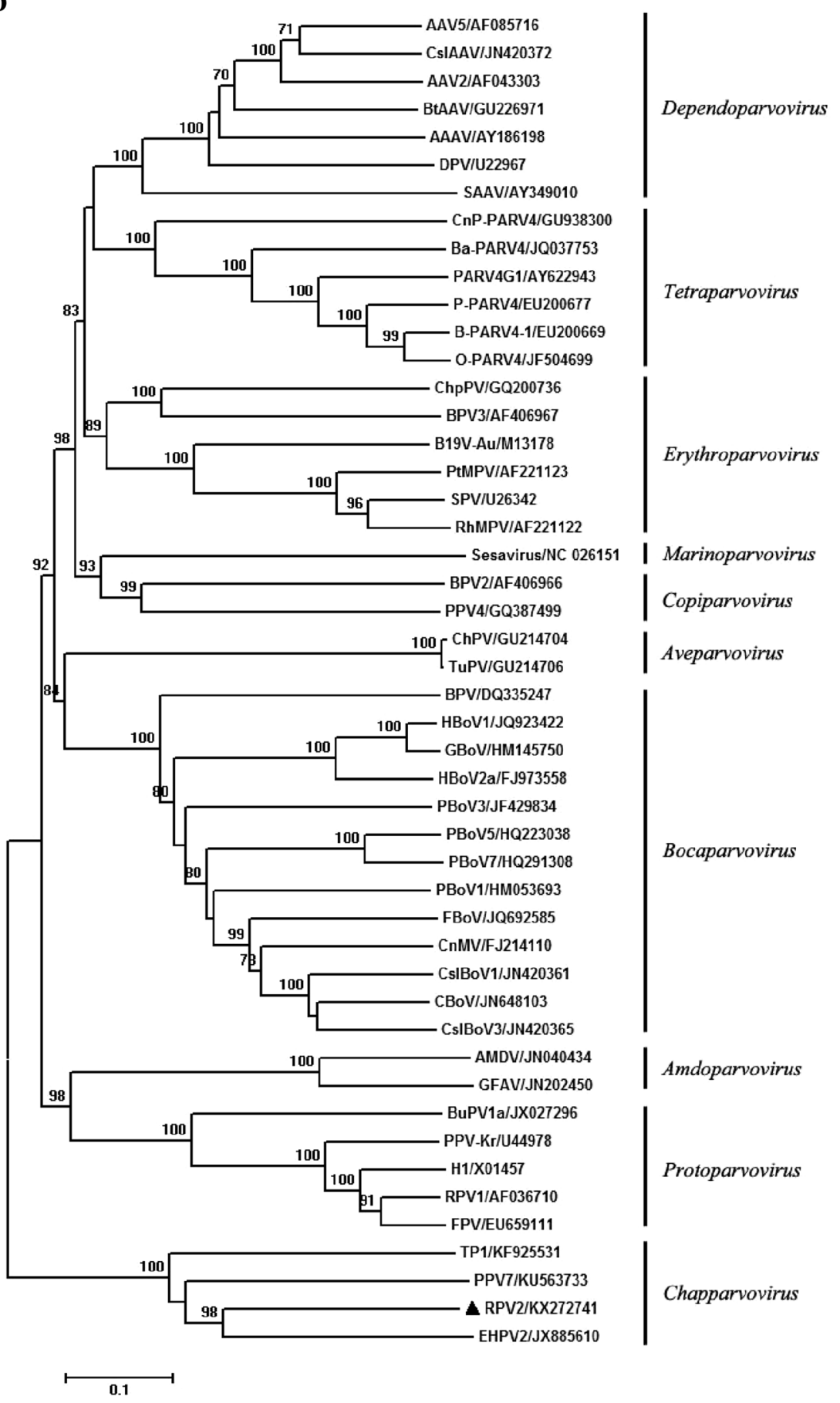

Fig. 1 (See legend on next page.) 
(See figure on previous page.)

Fig. 1 Genome organization and phylogenetic analysis of RPV2. a The genome rrganization of RPV2. The NS1 and VP proteins and the conservative ATP or GTP binding Walker loop motif are shown. b Phylogenetic tree was constructed over the NS1 amino acid sequences of 48 members in the Parvovirinae. The tree was derived using Neighbor-Joining analysis with 1000 bootstrap replicates. Scale bars indicate nucleotide substitutions per site, vertical bars represent the genera, and GenBank accession numbers are shown for the reference virus sequences, RPV2 identified in this study are marked with black triangle

Nextera XT DNA Sample Preparation Kit (Illumina) and sequenced using the Miseq Illumina platform with 250 bases paired ends with a distinct molecular tag for each pool. Resulting raw reads were trimmed for quality and primer, and de novo assembled into contigs. Sequences and contigs were compared to the GenBank non-redundant protein database using BLASTx with an E-value cutoff of $<10^{-5}$.

Results indicated there were 66 sequences reads showing the best BLASTx matches to EHPV2. The nearly complete genome was then determined by filling gaps by PCR and sequenced by Sanger method. The novel Chapparvovirus was designated rat parvovirus 2 (RPV2) and submitted to GenBank with accession no. KX272741. The near complete genome of RPV2 consists of 4222-nt and encodes two major proteins including the NS1 protein and VP protein, respectively. The NS1 protein was 654 amino acid in length possessing the ATP or GTP binding Walker loop motif ${ }^{306}$ GPSNTGKS ${ }^{313}$ (Fig. 1, panel a). Sequence analysis indicated that RPV2 NS1 shares the highest amino acid sequence identity of $51 \%$ with EHPV2. The VP protein was 472-aa in length and significantly shorter than other members of the subfamily Parvoviridae, which has an approximately 700-aa capsid protein. In addition, the conserve phospholipase $\mathrm{A}_{2}$ (PLA2) motif widely presented in the other members of the subfamily Parvoviridae is lack in RPV2 VP protein [11]. The RPV2 VP protein shares the highest amino acid sequence identity of $44 \%$ with PPV7. The ICTV states that parvoviruses sharing $>85 \%$ amino acid identity of NS1 protein belong to the same species. Based on the criteria, RPV2 identified here belongs to a novel species of Chapparvovirus.

To determine the relationship between RPV2 and the other members of the subfamily Parvoviridae, phylogenetic analysis over the NS1 protein of 48 members in the Parvovirinae was performed. Amino acid sequences of NS1 were aligned by ClUSTAL W, and phylogenetic trees were constructed with MEGA 6.0 software using the Neighbor-Joining statistical method based on the JonesTaylor-Thornton matrix-based model. Result showed that RPV2 clustered with EHPV2, PPV7, and TP1, forming a separate clade (Fig. 1, panel b). Among them, RPV2 has the closest relationship with EHPV2, which was identified from fecal samples of Eidolon helvum [7].

To investigate the prevalence of RPV2 in wild rats, viral nucleic acid was extracted from all the 40 individual samples using TaKaRa MiniBEST Universal Genomic DNA Extraction Kit Ver.5.0 (TaKaRa, Japan). A set of nested primers designed basing on the NS1 nucleotide sequence of RPV2 was used to perform PCR screening in the 40 rat fecal samples. Primer RPV2 F1 (5'TGGGTTCAGGCTACACAGGGTGG-3') and RPV2 R1 (5'-CAGTCGCCCCTCTCAGGGCT-3') were used for the first round of PCR, and RPV2 F2 (5'-AGCTG TACCCTGGGGAGAAATTGT-3') and RPV2 R2 (5'CGGGTTGTTGTCCTCTCGGCA-3') for the second round, the expected length of amplified fragment was $419 \mathrm{bp}$. Our results indicated $17.5 \%(7 / 40)$ of the fecal samples were positive. The PCR amplicons were T-A cloned and sequenced by Sanger method. Sequence analysis showed that the seven amplicons shared $>98 \% \mathrm{nu}-$ cleotide identity, indicating a single virus strain was prevalent in the wild rats in this area.

\section{Conclusions}

In summary, we detected a novel rodent Chapparvovirus in wild rats and characterized its nearly complete genome. Phylogenetic analysis indicated that the RPV2 clustered with EHPV2, PPV7, and TP1, forming a separate clade. According to the genetic distance-based criteria, RPV2 belonged to a novel species of Chapparvovirus. Our epidemiologic data indicated RPV2 was prevalent in the wild rats in this area.

\section{Abbreviations \\ BLAST, basic local alignment search tool; DPBS, Dulbecco's phosphate buffered saline; EHPV2, eidolon helvum parvovirus 2; NS1, nonstructural protein 1; ORF, open reading frame; PCR, polymerase chain reaction; PLA2, phospholipase $\mathrm{A}_{2}$; PPV7, porcine parvovirus 7; RPV2, rat Chapparvovirus 2; VP, capsid protein}

\section{Acknowledgement}

This work was supported by the Natural Science Foundation of Jiangsu Province No. BK20130502 and BK20140537, the National Natural Science Foundation of China No. 31302107, the Professional Research Foundation for Advanced Talents of Jiangsu University No. 12JDG085 and 13JDG087, the Postdoctoral Foundation of Jiangsu Province No. 1302058C and 1302057C, the China Postdoctoral Foundation No. 2014 M561597 and China Postdoctoral Special Foundation No. 2015 T80503.

\section{Authors' contributions}

WZ and SY conceived the study. SY and ZL performed all the experiments. SY wrote the paper. All authors read and approved the final manuscript.

\section{Competing interests}

The authors declare that they have no competing interests.

Ethics approval and consent to participate

Not applicable. 


\section{Author details}

'School of Medicine, Jiangsu University, Zhenjiang, Jiangsu 212013, People's Republic of China. ${ }^{2}$ School of Basic Medical Sciences, Ningxia Medical University, Yinchuan, Gansu 750000, People's Republic of China. ${ }^{3}$ School of Medicine, Jiangsu University, 301 Xuefu Road, Zhenjiang, Jiangsu 200240, People's Republic of China.

Received: 16 June 2016 Accepted: 25 July 2016

\section{Published online: 29 July 2016}

\section{References}

1. Conteville LC, Zanella L, Marin MA, Filippis AM, Nogueira RM, Vicente AC, Mendonca MC. Parvovirus B19 1A complete genome from a fatal case in Brazil. Mem Inst Oswaldo Cruz. 2015;110:820-1.

2. Perez $R$, Calleros $L$, Marandino A, Sarute N, Iraola G, Grecco S, Blanc $H_{\text {, }}$ Vignuzzi M, Isakov O, Shomron N, et al. Phylogenetic and genome-wide deep-sequencing analyses of canine parvovirus reveal co-infection with field variants and emergence of a recent recombinant strain. PLoS One. 2014:9:e111779.

3. Miranda C, Parrish CR, Thompson G. Canine parvovirus $2 \mathrm{c}$ infection in a cat with severe clinical disease. J Vet Diagn Invest. 2014;26:462-4.

4. Cotmore SF, Agbandje-McKenna M, Chiorini JA, Mukha DV, Pintel DJ, Qiu J, Soderlund-Venermo M, Tattersall P, Tijssen P, Gatherer D, Davison AJ. The family Parvoviridae. Arch Virol. 2014;159:1239-47.

5. Phan TG, Gulland F, Simeone C, Deng X, Delwart E. Sesavirus: prototype of a new parvovirus genus in feces of a sea lion. Virus Genes. 2015:50:134-6.

6. Palinski RM, Mitra N, Hause BM. Discovery of a novel Parvovirinae virus, porcine parvovirus 7, by metagenomic sequencing of porcine rectal swabs. Virus Genes. 2016;52:564-7.

7. Baker KS, Leggett RM, Bexfield NH, Alston M, Daly G, Todd S, Tachedjian M, Holmes CE, Crameri S, Wang LF, et al. Metagenomic study of the viruses of African straw-coloured fruit bats: detection of a chiropteran poxvirus and isolation of a novel adenovirus. Virology. 2013:441:95-106.

8. Reuter G, Boros A, Delwart E, Pankovics P. Novel circular single-stranded DNA virus from turkey faeces. Arch Virol. 2014;159:2161-4.

9. Zhang W, Li L, Deng X, Kapusinszky B, Delwart E. What is for dinner? Viral metagenomics of US store bought beef, pork, and chicken. Virology. 2014;468-470:303-10.

10. Zhang W, Li L, Deng X, Kapusinszky B, Pesavento PA, Delwart E. Faecal virome of cats in an animal shelter. J Gen Virol. 2014;95:2553-64.

11. Kemenesi G, Dallos B, Gorfol T, Estok P, Boldogh S, Kurucz K, Oldal M, Marton S, Banyai K, Jakab F. Genetic diversity and recombination within bufaviruses: Detection of a novel strain in Hungarian bats. Infect Genet Evol. 2015;33:288-92.

\section{Submit your next manuscript to BioMed Central and we will help you at every step:}

- We accept pre-submission inquiries

- Our selector tool helps you to find the most relevant journal

- We provide round the clock customer support

- Convenient online submission

- Thorough peer review

- Inclusion in PubMed and all major indexing services

- Maximum visibility for your research

Submit your manuscript at www biomedcentral.com/submit

) Biomed Central 